\title{
ABSORPTION AND EMISSION OF EUV RADIATION BY THE LOCAL ISM
}

\section{Francesco Paresce}

Space Telescope Science Institute, Homewood Campus, The Johns Hopkins University, Baltimore, MD 21218, USA and Astrophysics Division, Space Science Department, ESTEC, ESA.

\section{ABSTRACT}

The Berkeley EUV telescope flown on the Apollo Soyuz mission in July, 1975 established the existence of a measurable flux of EUV radiation $(100 \leq \lambda$ $\leq 1000 \AA$ ) originating from sources outside the solar system. White dwarfs, $\bar{f}$ lare stars and cataclysmic variables were discovered to be relatively intense compact sources of EUV photons. Moreover, this and other subsequent experiments have strongly suggested the presence of a truly diffuse component of the EUV radiation $f$ ield possibly due to thermal emission from hot $\left(\mathrm{T}>10^{5} \mathrm{~K}\right)$ interstellar gas located in the general vicinity of the sun $(r=100$ parsecs.) Closer to the HI, $912 \AA$ edge, the effect of a few hot 0 and $B$ stars has been shown to be very important in establishing the interstellar flux density. All these results imply that the local ISM is immersed in a nonnegligible EUV radiation field which, because of the strong coupling between EUV photons and matter, will play a crucial role in determining its physical structure. Conversely, of course, the local ISM is expected to leave a strong imprint on the EUV field reaching the terrestrial observer.

The objective of this review will be to assemble and critically analyze the available information on the local ISM derived from the limited EUV observations carried out so far. These include measurements of the spectra of bright EUV sources that reveal clear evidence of $\mathrm{H}$ photo absorption at $\lambda>400 \AA$ and of the He ionization edge at $228 \AA$. The EUV diffuse background is found to convey interesting information on the density, temperature and possible location of the hot ISM component that can be profitably compared with similar data on far uv absorption lines, principally OVI. The results discussed in this context will be shown to be quite useful in illuminating the path that future EUV sensitive observatories such as EUVE and Columbus might profitably follow in the near future.

\section{INTRODUCTION}

The extreme ultraviolet (EUV) band of the electromagnetic spectrum extending approximately from 1000 to $100 \AA$ should, in principle, be perfectly suited to the detailed study of the local interstellar medium (LISM). The high absorptivity of matter in the EUV insures a high probability of lightmatter interaction over relatively short, well defined path lengths and the availability of a large number of well studied permitted transitions and spectral features provides the tools required to accurately probe a wide range of ISM materials and configurations. The effective photoionization cross section of the LISM, $\sigma_{e}=\Sigma n_{i} \sigma_{i} / n_{H}$ where $n_{i}$ and $n_{H}$ are the volume densities of 
element $i$ and $H$, respectively for a variety of possible phases has been computed recently by Cruddace et al, 1974. A peak cross section of almost $10^{-17} \mathrm{~cm}^{2}$ at $912 \AA$ followed by a $u^{-3}$ decline to shorter wavelengths

superimposed more or less prominently on which are the discontinuities due to the ionization edges of heavier elements are the key features of these calculations.

Using these results, the distance at which $90 \%$ of the radiation emitted by an EUV source is absorbed in a neutral LISM phase is plotted in Figure 1 as a function of wavelength for $n_{H}$ varying between 0.2 and $0.03 \mathrm{~cm}^{-3}$, a range consistent with possible scenarios within the first hundred parsecs or so of the sun. This figure clearly shows that for all intents and purposes, the observable EUV source horizon is limited to a few hundred parsecs at the very most at the wavelengths where most of the absorption is occurring. Thus, we can expect to probe accurately the ISM only within this local region by measuring the EUV spectrum of any compact or diffuse sources Nature can provide. This, of course, assumes a decent understanding of the sources' inherent emission spectrum can be obtained independently by some other means. In the following, I describe the progress made so far in this very new and exciting field and discuss some of the possibilities for future work.

\section{COMPACT SOURCES}

To move from principles to reality, we need observable EUV sources. Up to 1975 these were mainly gleams in a few scientists' eyes. Since then, thanks mainly to the pioneering observations carried with the EUV grazing incidence telescope on the ASTP, the situation has improved dramatically. The compact sources discovered so far are listed in Table I with their positions in the sky, distance, type, and approximate flux at Earth in the wavelength bands in which they were detected. The reference to the discovery paper for the appropriate source is listed in the last column. A more detailed description of the discovery and characteristics of most of these sources can be found in Paresce, 1980.

Several points about the present source statistics should be emphasized. First, the sources observed so far all fall within 100 parsecs of the sun although distances are somewhat uncertain, possibly up to a factor of two in some cases. Second, only 7 sources in all have been observed so far with, moreover, only four that can be considered bone fide EUV sources. The cataclysmic variables are more aptly described as soft $x$-ray sources and visible only at $\simeq 100 \AA$ and in outburst (Holberg, 1984). Third, the "true" EUV sources are all DA White Dwarfs. Fourth, only HZ43 has been observed throughout the EUV from $\approx 800$ to $100 \AA$. Its spectrum between $\sim 200$ and $2000 \AA$ is shown in Figure 2, reprinted here by permission from J. Holberg. The solid line represents the expectations of an unblanketed, pure hydrogen, non-LTE model atmosphere with $\mathrm{T}_{e}=55000 \mathrm{~K}, \log \mathrm{g}=8$ absorbed by an LISM of density $\mathrm{n}_{\mathrm{H}}=0.002 \mathrm{~cm}^{-3}$ with the dashed 1 ines quantifying the effects of changing $\mathrm{n}_{\mathrm{H}}$ by $\pm 50 \%$.

It is evident from the results displayed in Figure 2 that the $500-800 \AA$ region of the spectrum is the most sensitive to variations in $n_{H}$ as expected, 


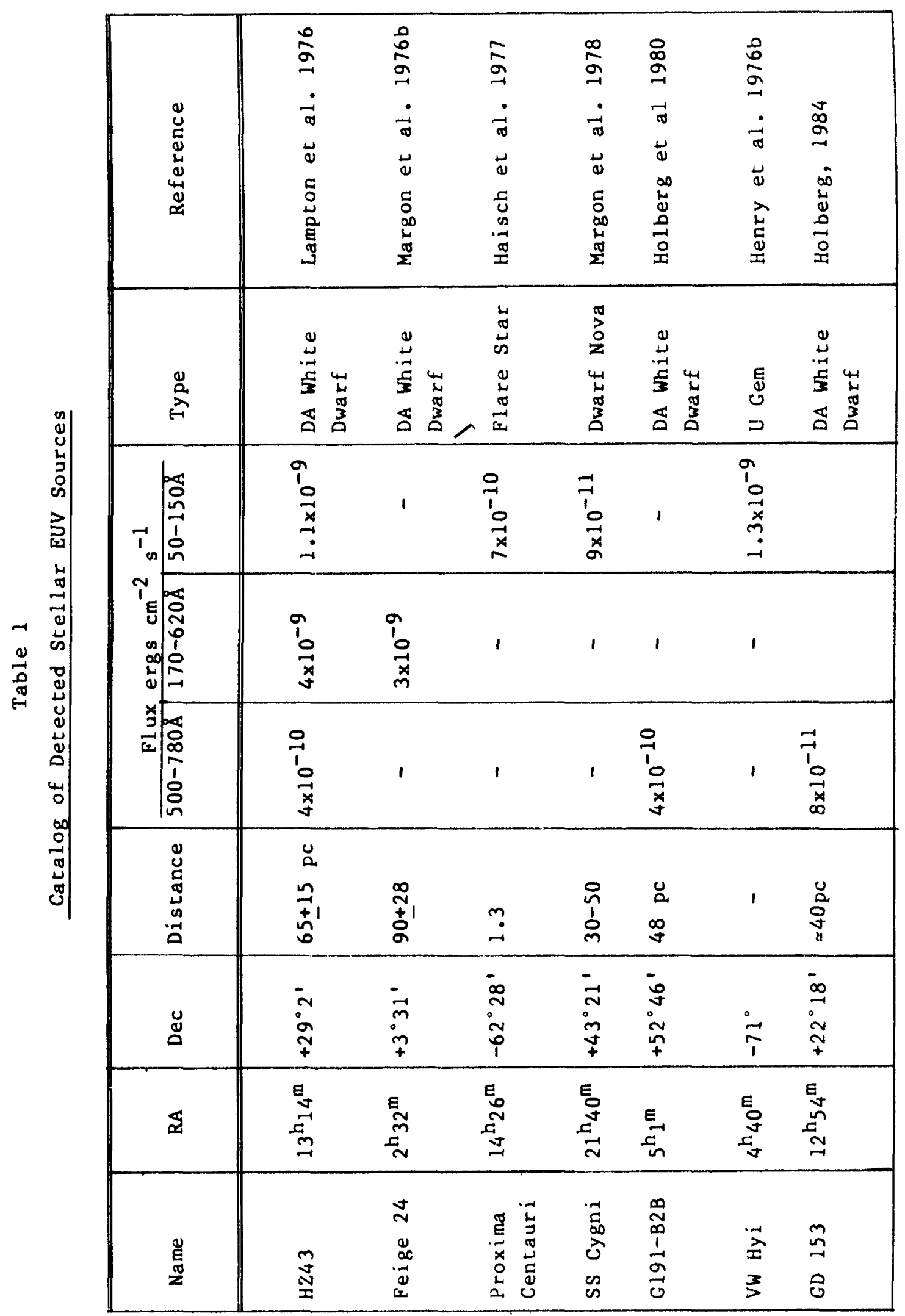

171 
and that the theoretical model fails to account for the detailed structure of the spectrum in this band and for any of the observed data in the region below $\approx 500 \AA$. This case well illustrates the difficulties associated with accurate extraction of LISM information from complicated stellar emission patterns. Wesemael et al., 1980, and Wesemael, 1981 have computed the expected EUV emission from hot, high gravity stars with pure $H$ or He atmospheres that can be used to compare with observations. The shape of the unabsorbed inherent stellar spectrum above $\approx 400 \AA$ for these objects depends critically on the effective temperature $T_{e}$, the surface gravity $g$ and the chemical composition of the surface. In order to reproduce a discontinuity of a factor of $\approx 2$ at the $\mathrm{He}^{+}$ionization edge at $228 \AA$, Malina et al., 1982 resorted to pure hydrogen models having a trace amount of helium $\left(\sim 6 \cdot 10^{-5}\right.$ fractional number density of hydrogen). The Malina et al. model, however, still fails to account for the soft $x$-ray and far uv spectrum. The large uncertainties in the stellar models clearly influence the determinations of the LISM parameters since the former must be well known before precise inferences can be drawn on the latter. This problem becomes particularly acute when observational upper limits to EUV emission features whose origins are extremely controversial and uncertain are used to draw conclusions on the LISM as has been attempted recently with Capella and the He II, $304 \AA$ line by Bobroff et al., 1984.

Armed with a better appreciation of the difficulties involved with this brief introduction, we can understand the results of the attempts made so far to derive the neutral hydrogen column density towards EUV sources 1 isted in Table 2. The best studied source, HZ43, shows how dramatically this parameter can vary with the sophistication of the stellar model employed and, to some degree, on the quality of the data obtained. Thus, we see the log of the $H$ column dropping from a high of 18.6 when a black body (BB) model at $\mathrm{T}_{\mathbf{e}}=$ $110000 \mathrm{~K}$ was used to less than 17.3 when the Berkeley rocket experiment was analyzed in terms of a sophisticated mixed chemical composition model. The variation is not trivial since it implies a change of the average hydrogen volume density from $2 \cdot 10^{-2}$ to less than $10^{-3} \mathrm{~cm}^{-3}$. Although it is pretty well established that $n_{H}$ cannot be larger than $\approx 0.01 \mathrm{~cm}^{-3}$ towards $H Z 43$ without violating observational constraints in the visible (Auer and Shipman, 1977), any stronger statement might not be appropriate at the present time in view of the still large uncertainties in the correct stellar model and even in the observations themselves. Contamination due to contributions from the second order of the Voyager grating have been invoked to explain part of the signals above $500 \AA$ seen in the Voyager data in Figure 2 and the discontinuity at 228 $\AA$ has not been confirmed by EXOSAT. These are all troubling questions that need to be securely resolved before more progress can be made.

Nevertheless, the results reported in Table 2 do have important implications for the LISM. As discussed in more detail by Paresce, 1984 most of the EUV determinations of $N(H I)$ except perhaps G191-B2B lie well below the column density expected at their distance from an extrapolation of a very local medium having $\mathrm{n}_{\mathrm{H}} \simeq 0.07 \mathrm{~cm}^{-3}$. This discrepancy is particularly striking in the case of $\mathrm{HZ43}$ at $1=50^{\circ}, \mathrm{b}=84^{\circ}$ where it reaches at least an order of magnitude. Since GD153 is only $8^{\circ}$ away from $\mathrm{HZ} 43$ and at a similar distance it is comforting that its implied column density is consistent with HZ43. A lower than average density implies that the very local cloud described by 
TABLE 2

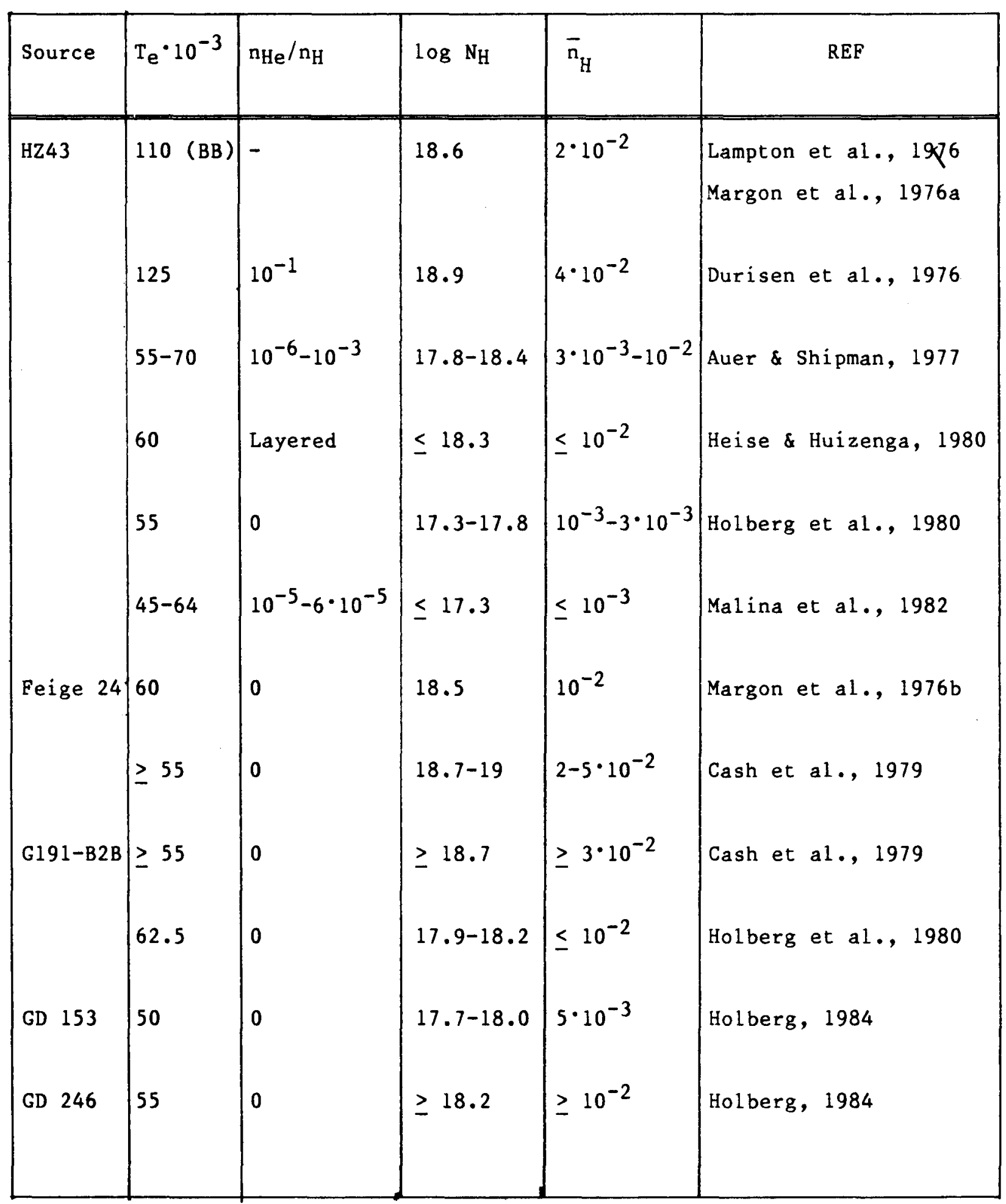


Bruhweiler (1982) can only be a few parsecs in extent outside the galactic plane. If the discrepancy between Cash et al., 1979 and the Voyager results on G191-B2B could be settled, this object might resolve the important issue concerning the extent of the local cloud in the plane itself, since it lies at $1=156^{\circ}, b=7^{\circ}$.

A correlated issue in this context is the extent of the ionization cavity around the very hot white dwarfs used in the survey summarized in Table II. As first pointed out by Hills, 1972 and further developed by Dupree and Raymond, 1983, cavities of many parsecs in extent may be carved out of the ISM if the $\mathrm{H}$ density is of order $0.01-0.1 \mathrm{~cm}^{-3}$. Feige 24 shows clear evidence for such an extended $H$ II region. Consequently, if the cavity radius is a fair fraction of its distance, transforming the measured column density into an average volume density over the whole distance might lead to an underestimate of the actual density of the absorbing medium. The effect would be most critical for very nearby objects and probably does not significantly affect the results shown in Table II but might have to be accounted for in the future when more white dwarfs will, hopefully, be detected by ROSAT, EUVE and Columbus / FUSE.

A way out of this problem is to use the EUV emission from hot transition regions and coronae of nearby cool stars. The expected emission from these sources have been computed by Landini et al., 1984 using a self-consistent formulation of a magnetically confined coronal loop model. The effect of even a small amount of absorption is quite dramatic and should be exploited. The crucial advantages of using such sources are their very high space density and their better understood spectrum as it is very similar to the sun's. The advantage of using white dwarfs, on the other hand, is their better defined continuum and relative absence of emission lines which may hamper absorption work. An interstellar $\mathrm{He}^{+}$ionization edge, for example, could be separated from a purely stellar one by even moderate resolution $(\lambda / \Delta \lambda>100)$ instrumentation as is being presently comtemplated for EUVE and Columbus/FUSE.

\section{THE DIFFUSE COMPONENT}

Between $\simeq 2$ and $10 \mathrm{keV}$, the intensity of the $x$-ray background exhibits a power law dependence with photon energy and appears essentially isotropic to moderate angular resolutions surveys. Possible sources of this emission are discussed by Marshall et al., 1980 and Fabian, 1981. Below $2 \mathrm{keV}$, however, the diffuse background becomes decidedly anisotropic and deviates sharply from the extrapolation of the higher energy spectrum even in the absence of hydrogen absorption as shown in Figure 3. The spectrum continues to climb well into the EUV, peaking at 4-10 photons $\mathrm{cm}^{-2} \mathrm{~s}^{-1} \mathrm{eV}^{-1}$ at $\approx 200 \AA$ before appreciable ISM absorption sets in. The background fluxes plotted in Figure 3 are described in Paresce and Stern, 1981 and represent average intensities in regions of the sky far from the established soft $x$-ray enhancements due to emission from much higher temperature gas (see McCammon et al., 1983 and Marshall and Clark, 1984 for a review of soft x-ray survey results). Although 
there is evidence for a substantial ( $20 \%)$ contribution due to unresolvedpoint sources above $\approx 250 \mathrm{eV}$, in the EUV the radiation field is almost certainly truly diffuse in origin (Stern and Bowyer, 1980; Rosner et al., 1981).

The failure of any known non-thermal emission mecharism to account for the observed spatial variations and the average spectrum shown in Figure 3 , compelling evidence for the existence of hot $\left(T>10^{5} \mathrm{~K}\right.$ ) gas in the ISM from 0 VI absorption measurements and a supernova blast wave production mechanism at least theoretically identified and understood all together make a strong case for a thermal plasma origin of the EUV background. In this case, the intensity of the radiation field is given by:

$I(E)=\frac{1}{4 \pi} \int_{1} \int_{T} \phi(E, T) n_{e c} \frac{d n_{e c}}{d \ln T} e^{-\tau(E)} d l d \ln T$

where $\tau(E)=\sigma(E) N(H I)$ is the optical depth of the absorbing medium, $\phi(E, T) n_{e c}{ }^{2}$ is the volume emissivity of the gas at $E$ and $T$, $n_{e c}$ the electron density of the emitting cloud of gas and 1 is the distance parameter along the line of sight. Unfortunately, eq. 1) is not invertible easily unless some simplifying assumptions are introduced. The most common is that the bulk of the emission is due to a single temperature component. Although there is no a-priori theoretical reason to suppose the gas is thermostatted precisely at one temperature, observations are normally made with instruments that are sensitive only to a rather narrow range of photon energies. Moreover, the temperature distribution around a classical evaporating cloud is very sharply peaked around the typical substrate temperature (McKee and Cowie, 1977).

Having thus disposed momentarily of the inhomogeneities in the physical structure of the emitting plasma, its spatial distribution has still to be dealt with. Not having any knowledge of this parameter it is common practice to consider two limiting extreme cases. The first assumes the emitting plasma is homogeneously mixed or finely interspersed with the absorbing medium. In this case, it can be shown that eq. 1 reduces to:

$I(E)=\frac{1}{4 \pi} \phi(E, T) \frac{<e_{e^{>}}}{\sigma_{e}{ }^{<n_{H}>}}(1-\exp [-\sigma(E) N(H I)])$

where $\left\langle n_{e}\right\rangle=f n_{e c}$ and $f$ is the filling factor of the hot plasma and $\left\langle n_{H}\right\rangle$ is the average neutral hydrogen density in the line of sight. In this scenario, the observations shown in Figure 3 can be fitted by models parameterized by the variables $\left\langle n_{e}\right\rangle^{2} /\left\langle n_{H}\right\rangle, T$ and $N(H I)$.

In the other extreme we assume that the emission from the hot plasma is absorbed by a single cool slab of material of column density N(HI). Then the intensity $I(E)$ can be expressed simply as:

$I(E)=\frac{1}{4 \pi} \phi(E, T)<n_{e}^{2}>R \exp [-\sigma(E) N(H I)]$ 
where $R$ is the physical length of the emitting region. In this case, the theoretical expectations may be parameterized by N(HI), T and the emission measure EM of the gas:

$E M=\int n_{e}^{2} d r=\left\langle n_{e}^{2}\right\rangle R=n_{e c}^{2} f R=\frac{(p / k)^{2}}{(1.91 T)^{2}} f R$

The actual spatial distribution clearly lies somewhere between these extreme cases. For $\tau<0.5$, in fact, the interspersed plasma model becomes, for all intents and purposes, indistinguishable from the slab absorbed model with $N(H I)=0$. Finally, we can relax the isothermal assumption adopted so far by allowing the emitting plasma to have a specified distribution of temperatures for each of the two cases considered above. This effect was first explored by Paresce and Stern (1981) who adopted the power law form suggested by Jenkins $(1978 b)$ :

$\frac{d n_{e}}{d \ln T}=c_{e} T^{\alpha}$

where $\mathrm{C}_{e}$ is an independently determined normalization parameter and $\alpha$ is the power law index. In this more complicated scenario, the theoretical slab absorbed model for example depends on $\alpha, N(H I)$, EM, and the temperature limits of integration in eq. $1, \mathrm{~T}_{1}$ and $\mathrm{T}_{2}$.

Computations of the plasma emissivity $\phi(E, T)$ have been carried out by a number of authors in the last few years. Only very recently, however, has the full 1 to $2000 \AA$ range of the spectrum been covered systematically (see Gaetz and Salpeter, 1983, Paresce et al,, 1983 and references therein for more complete discussion). The expected emission spectrum in photons $\mathrm{cm}^{-2} \mathrm{~s}^{-1}$ $\mathrm{sr}^{-1} \AA^{-1}$ of a plasma at $\mathrm{T}=2 \cdot 10^{5} \mathrm{~K}, E M=0.1 \mathrm{~cm}^{-6}$ pc absorbed by a slab of $N(H I)=2 \cdot 10^{19} \mathrm{~cm}^{-2}$ is shown in Figure 4 as a function of wavelength. The main assumption that has to be made to generate expected fluxes such as those shown is that the gas is in steady state collisional ionization equilibrium. If the plasma has been recently subjected to shock heating such an assumption may be naive and we should expect substantial deviations for some if not all of the emission features in the EUV and far UV displayed in this figure.

Detailed comparison with observations, however, come so close to self consistency that this desperation move with its huge associated uncertainties may not actually be required at least not with the quality of the data presently available. The situation for the EUV and far UV has been discussed in some detail by Paresce and Stern, 1981; Paresce et al., 1983 and Kimble, 1983. In general, the observations down to $100 \AA$ and perhaps slightly beyond to the $C$ soft $x$-ray band çan be reasonably well explained by emission from a relatively nearby hot $\left(10^{5} \leq \mathrm{T}<10^{6} \mathrm{~K}\right)$ plasma that pervades a fair fraction of space out to $\approx 100$ pc or so. More specifically, if the simple slab absorbed single tenperature model comes closest to reality, the range of the three free parameters allowed by the observations is given by the regions bounded by the 
solid lines in Figure 5 . These curves are the $90 \%$ confidence level contours obtained by using a minimum $x^{2}$ fitting procedure with three degrees of freedom.

Several conclusions leap out from even a cursory glance at Figure 5 . First, the temperature of the hot gas cannot be much higher than $\approx 5 \cdot 10^{5} \mathrm{~K}$. Second, there cannot be much more than $\times 10^{20}$ absorbing $H$ atoms in the way and, third, the temperature cannot be much lower than $2.5 \cdot 10^{5} \mathrm{~K}$ if we impose an upper limit on the gas pressure of $\mathrm{p} / \mathrm{k}=10^{4} \mathrm{~cm}^{-3} \mathrm{~K}$ as shown by the dotted line in Figure 5 for a fixed effective path of 100 parsecs. Using the interspersed models reduces these ranges even further. The real problem with isothermal models, however, apart from the absence of a known physical mechanism that would thermostat the plasma in a region of high cooling rates, is that they imply far too much $O$ VI absorbing gas. If we demand, as we should, consistency with the 0 VI results summarized by Jenkins $(1978 a$, b) we are forced to look for another source of the EUV background or require a significant pressure imbalance in the ISM, evidence for which has yet to surface. Notice that there is no easy escape from this conclusion here as there is in the soft $x$-rays since the preferred temperatures at which a plasma emits in the EUV is precisely the same as the one at which the $0^{+5}$ ion is most comfortable.

The temperature distribution models represented by eq. 5 go a long way towards easing this dilemma, however. The allowed parameter space for this case and the absorbing slab scenario is shown in Figure 6 for $\log \mathrm{T}_{1}=5.0$ and $\log \mathrm{T}_{2}=5.8$. Allowing for the customary factor of two uncertainty both in the predictions and the gbseryations, a model of this type having $f=0.4$, $\mathrm{R}=100 \mathrm{pc}, \mathrm{p} / \mathrm{k}=1.5 \cdot 10^{4} \mathrm{~cm}^{-3} \mathrm{~K}, \alpha=1.5$ reconciles all the available observations and theory in a self consistent manner. The predicted average $0^{+5}$ ion density is $1.2 \cdot 10^{-7} \mathrm{~cm}^{-3}$ over this pathlength which, although is $\approx$ a factor of four higher than the average of $3 \cdot 10^{-8} \mathrm{~cm}^{-3}$ over kpc pathlengths, is quite consistent with the more local lines of sight (Jenkins, 1978a). We note in passing that the EUV background compatible value of $\langle n(0$ VI $)\rangle$ is much more consistent with that predicted by the McKee and Ostriker (1977) theory to be present in the conduction interfaces.

As Figure 4 clearly illustrates, if we assume the EUy background is generated by a hot plasma in the $T>10^{5} \mathrm{~K}, \mathrm{EM}=10^{-1} \mathrm{~cm}^{-6} \mathrm{pc}$ range, a substantial part of the total emission has to show up in the 912 to $1600 \AA$ band. The expected fluxes may have been detected towards the north galactic pole by Feldman et al., 1981 but an interpretation of these observations by Paresce et al., 1983 in terms of a hot plasma emission model yields only a marginal compatibility with the EUV emitting plasma. Since the lines in the far UV are all of lower ionization stages, sufficient emissivity is achieved at generally lower temperatures at comparable emission measures. The tail of the best fit EUV power law distribution is insufficient to account for the intensity of the observed EUV 1ines. In the absence of additional data on this fascinating subject, one must conclude that the present observations may indicate either the presence of a new cooler component of the local ISM or that the assumption of a steady state ionization equilibrium needs to be 
revisited. Maneuvering room in the steady state isothermal models is very limited, unfortunately since upper limits on $\mathrm{N} V$ place severe constraints on filling factors of any gas at temperatures around $2 \cdot 10^{5} \mathrm{~K}$.

Similarly interesting are the observations in the 900-1200 $\&$ region summarized by Kimble, 1983. In this range, the density of the radiation field is dominated by emission of known normal stars. The observational upper limits to the specific intensity of the radiation field in particular directions available presently are just beginning to constrain the allowed regions of the physical characteristics of a hot plasma. If the upper limits to the 912-1200 A radiation field intensity obtained recently by the ECOM-721 spectrometer on the STP78-1 satellite that surveyed an appreciable fraction of the sky where the contribution of known sources is negligible are converted by means of equations $1-5$ into a limit on emission measure as a function of $T$, for example, the allowed region of $F$ igure 5 is reduced to the $\log T \geq 5.4$, EM $\leq 0.1$ region of the plane. More sensitive observations made by Voyager 2 are now becoming available and a closer scrutiny of the implications of the measurements in this range deserves the highest priority. What has been definitely lacking up to now is a systematic study of the global pattern of the diffuse background from the soft $x$-rays through the EUV and well into the far UV. The theoretical underpinnings should now exist for a thorough study of the local hot gas with a more physically plausible distribution of temperatures and, perhaps, filling factors such as that suggested by eq. 5 .

\section{CONCLUSIONS}

We have established in this brief overview that the local ISM leaves a clear signature of its presence on the EUV spectra of nearby compact sources, on the spectrum and, probably, on the spatial variations of the diffuse EUV background. Compact objects such as hot white dwarfs and even main sequence stars certainly disturb the surrounding ISM by creating large ionization cavities and by contributing to the general radiation density at any point. The diffuse background whether truly diffuse or not also contributes to the ionization of the local ISM. The most efficient photons in this respect are those in the Lyman continuum. The observational situation in this region is presently very confused, unfortunately. A measurement of the intensity of the radiation field at $750 \AA$ in a particular direction has been claimed by Sandel et al., 1979 to be $2 \mathrm{~s}^{3} \cdot 10^{-8}$ ergs $\mathrm{cm}^{-2} \mathrm{~s}^{-1} \mathrm{sr}^{-1} \AA^{-1}$ or approximately 900 photons $\mathrm{cm}^{-2} \mathrm{~s}^{-1} \mathrm{sr}^{-1} \AA^{-1}$.

It is very instructive to consider what implications this flux averaged over the whole sky would have on our LISM. This problem has been studied by Meier, 1980 and Kimble, 1983. In a steady state situation where recombination just balances photoionization, a local Hydrogen ionization fraction of 0.5 or or less. Since 0.5 is a reasonable observational upper limit set by solar backscatter observations, the local flux cannot be much greater than this value. Conversely, if the Voyager field were actually as high as quoted, the LISM would have an ionization fraction of at least $\mathbf{0 . 9}$. The implication has to be that the Voyager measurement is not of a typical line of sight. A 
recent theoretical calculation performed by Grewing (1975) also predicts a radiation field that would ionize the LISM to a degree not observed in practice.

Consequently, it is in the 700-900 $\AA$ range that the LISM and the radiation field are most intimately coupled and where observations of one crucially implicate the other. It is safe to conclude that the EUV is one of the most promising and fertile areas of LISM research today and in the immediate future.

\section{REFERENCES}

Auer, L. H., and Shipman, H. L., 1977, Ap. J. (Lett), 211, L105. Bobroff, N., Nousek, J., and Garmire, G., 1984, Ap. J., $277,678$.

Bruhweiler, F., 1982, in "Advances in UV Astronomy: Four Years of IUE Research," NASA CP-2238, p. 125.

Cash, W., Bowyer, S., and Lampton, M., 1979, Astr. Ap., 80, 67.

Cruddace, R., Paresce, F., Bowyer, S., and Lampton, M., 1974, Ap. J., 187, 497.

Dupree, A. K., and Raymond, J. C., 1983, Ap. J. (Lett.), 275, L71.

Durisen, R. H., Savedoff, M. P., and Van Horn, H. M., 1976, Ap. J. (Lett.),

Fabian, A. C., 1981, in "Tenth Texas Symposium on Relativistic Astrophysics," eds. R. Ramaty and F. C. Jones, N.Y. Acad. Sci., 375, 235.

Feldman, P. D., Brune, W. H., and Henry, R. C., 1981, Ap. J., 249, L51.

Gaetz, T. J., and Salpeter, E. E., 1983, Ap. J. Suppl., 52, 155 .

Grewing, M., 1975, Astr. Ap., 38, 391.

Haisch, B., Linsky, J., Lampton, M., Paresce, F., Margon, B., and Stern, R., 1977, Ap. J. (Lett.), 213, L119.

Heise, J., and Huizenga, H., 1980, Astr. Ap., 84, 280.

Henry, P., Bowyer, S., Rapley, C. G., and Culhane, J. L., 1976b, Ap. J. (Lett.), 209, L29.

Hil1s, J. G., 1972 , Astr. Ap., 17, 155.

Holberg, J. B., Sandel, B. R., Forrester, W. T., Broadfoot, A. L., Shipman, H. L., and Barry, J. L., 1980, Ap. J. (Lett.), 242, L119.

Holberg, J. B., Forrester, W. T., and Broadfoot, A. L., 1980, Bull. AAS, 12, 872.

Holberg, J., 1984, these proceedings.

Jenkins, E. B., 1978a, Ap. J., 219, 845 .

Jenkins, E. B., 1978b, Ap. J., 220,107 .

Kimble, R. A., 1983, Ph.D. Dissertation, University of California, Berkeley.

Lampton, M., Margon, B., Paresce, F., Stern, R., and Bowyer, S., 1976, Ap. J. (Lett.), 203, L71.

Landini, M., Monsignori Fossi, B. C., Paresce, F., and Stern, R., 1984, Ap. J., in press.

Malina, R. F., Bowyer, S., and Basri, G., 1982, Ap. J., 262, 717.

Margon, B., Liebert, J., Gatewood, G., Lampton, M., Spinrad, H., and Bowyer, S., 1976a, Ap. J., 209, 525.

Margon, B., Lampton, M., Bowyer, S., Stern, R., and Paresce, F., 1976b, Ap. J. (Lett.), 210, L79. 
Margon, B., Szkody, P., Bowyer, S., Lampton, M., and Paresce, F., 1978, Ap. J., 224, 167 .

Marshall, F. E., Boldt, E. A., Holt, S. S., Miller, R. B., Mushotzky, R. F., Rose, L. A., Rothschild, R. E., and Serlemitsos, P. J., 1980, Ap. J., 235,4 .

Marsha11, F. J., and Clark, G. W., 1984, Ap. J., in press.

McCammon, D., Burrows, D. N., Sanders, W. T., and Kraushaar, W. L., 1983, Ap. J., 269, 107 .

Mckee, C. F., and Cowie, L., 1977, Ap. J., 215, 213.

McKee, C. F., and Ostriker, J. P., 1977, Ap. J., 218, 148.

Meier, R. R., 1980, Astr. Ap., 91, 62.

Paresce, F., 1980, in "Astrophysics from Spacelab," eds. P. L. Bernacca and R.

Ruffini, Astrophysics and Space Science Library, Reidel, 81, 243.

Paresce, F., and Stern, R., 1981, Ap. J., 247, 89.

Paresce, F., Monsignori Fossi, B. C., and Landini, M., 1983, Ap. J. (Lett.), 266, L107.

Paresce, F., 1984, A. J., 89, (7), July, 1984.

Rosner, R., Avni, Y., Bookbinder, J., Giacconi, R., Golub, L., Harnden, F., Maxson, C. W., Topka, K., and Vaiana, G. S., 1981, Ap. J. (Lett.), 249, L5.

Sande1, B. R., Shemansky, D. E., and Broadfoot, A. L., 1979, Ap. J., 227, 808. Stern, R., and Bowyer, S., 1980, Astr. Ap., 83, L1.

Wesemael, F., 1981, Ap. J. Suppl., 45, 177.

Wesemael, F., Auer, L. H., Van Horn, H. M., and Savedoff, M. P., 1980, Ap. J. Suppl., 43, 159 . 


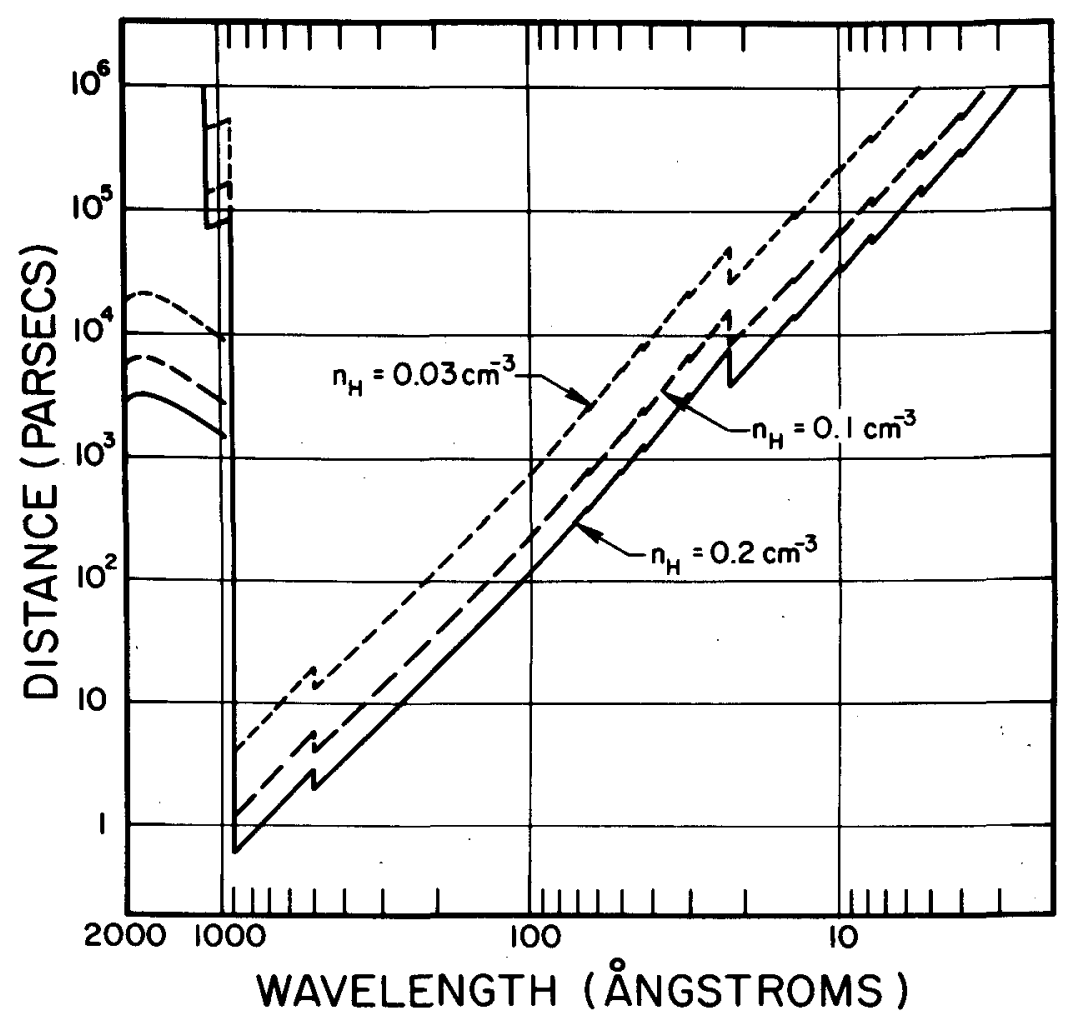

Figure 1

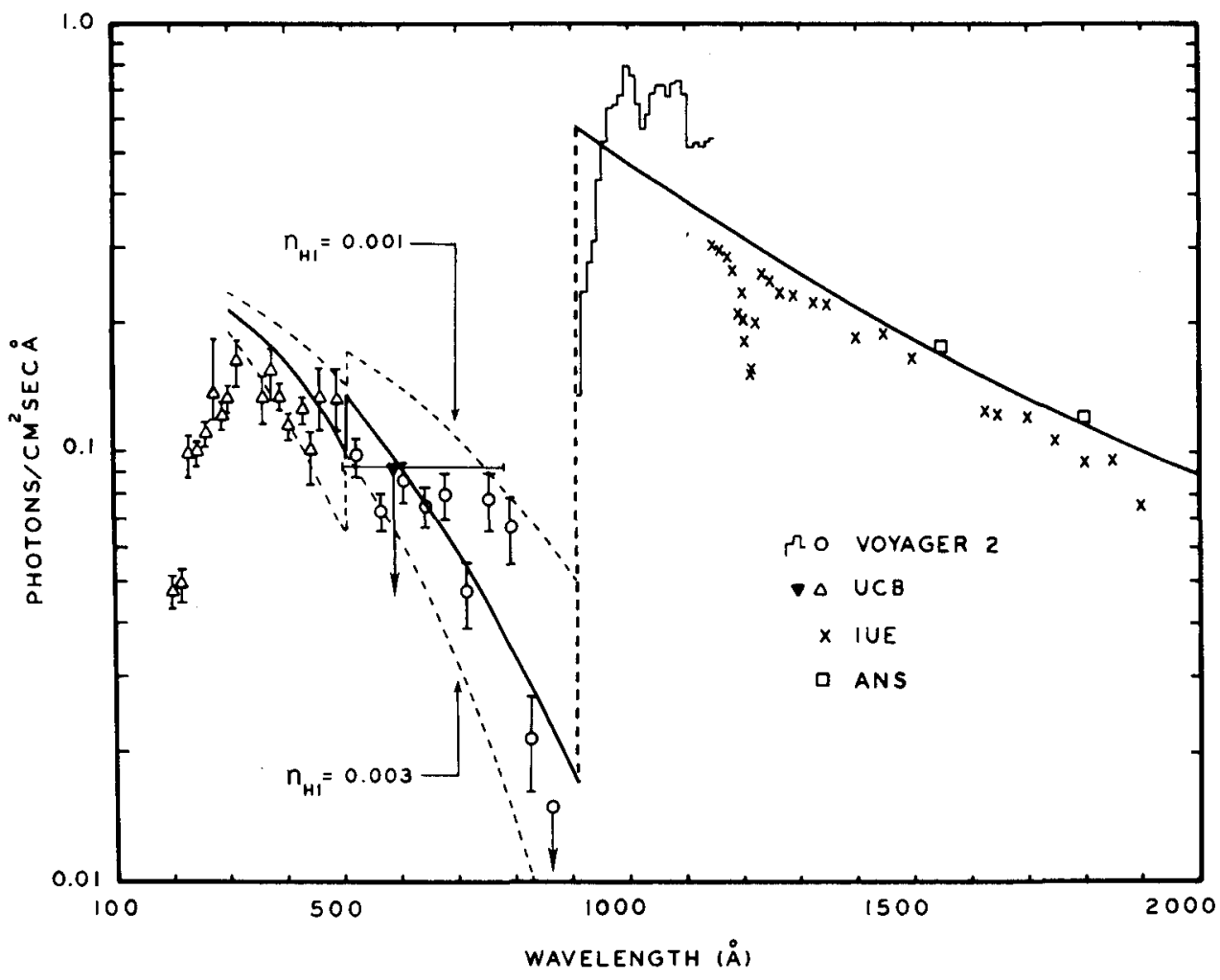

Figure 2 


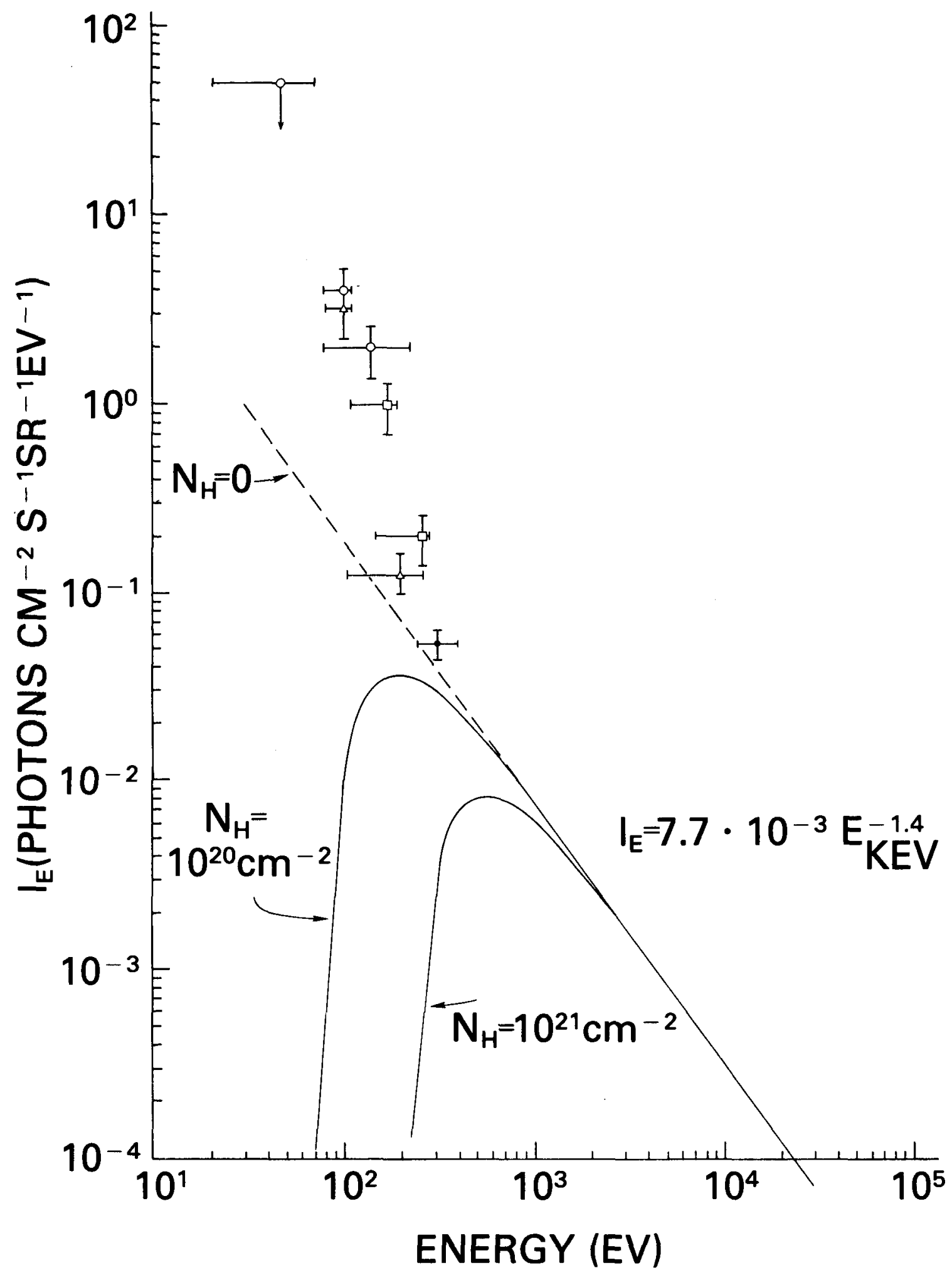

Figure 3 


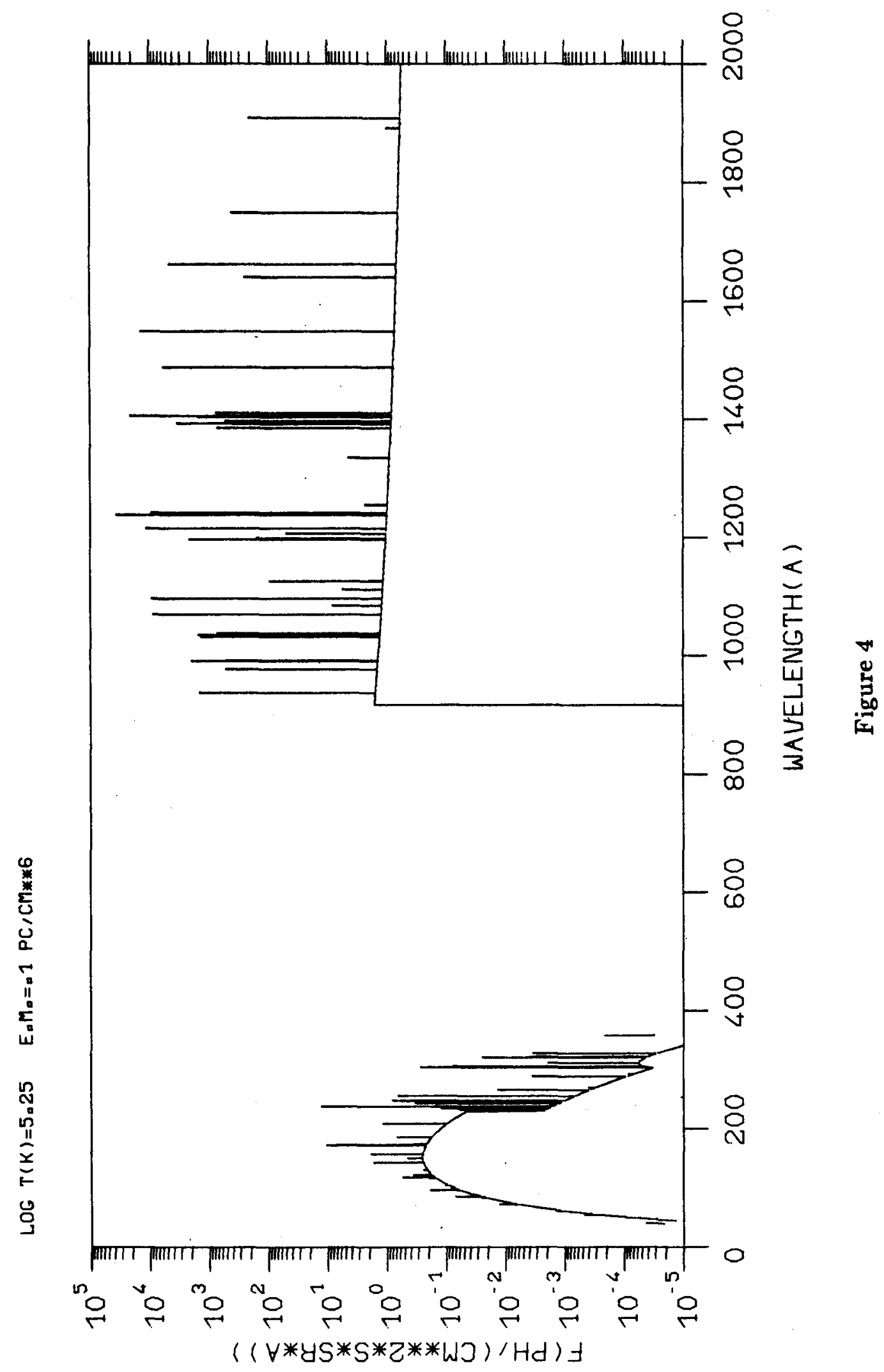




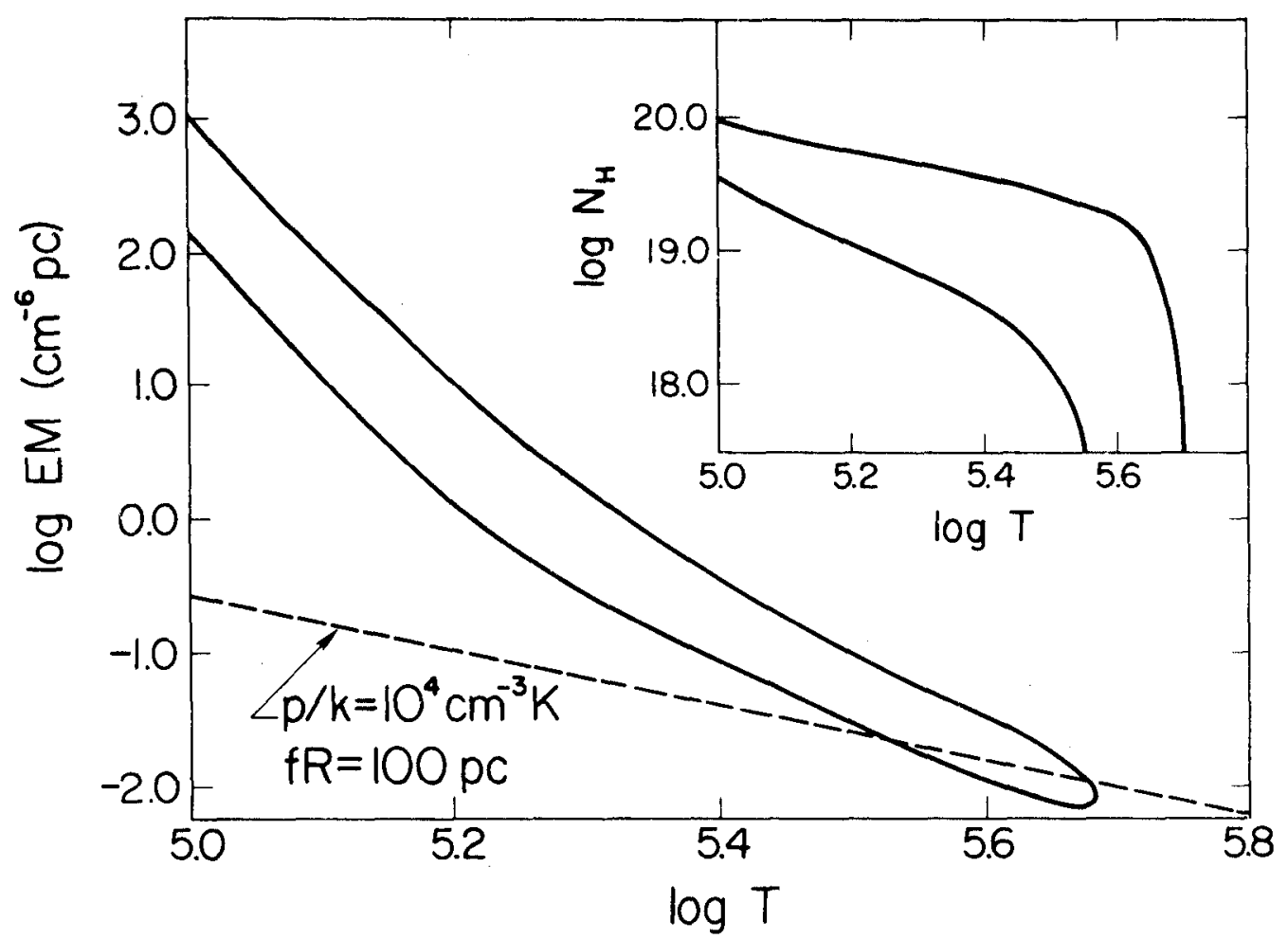

Figure 5

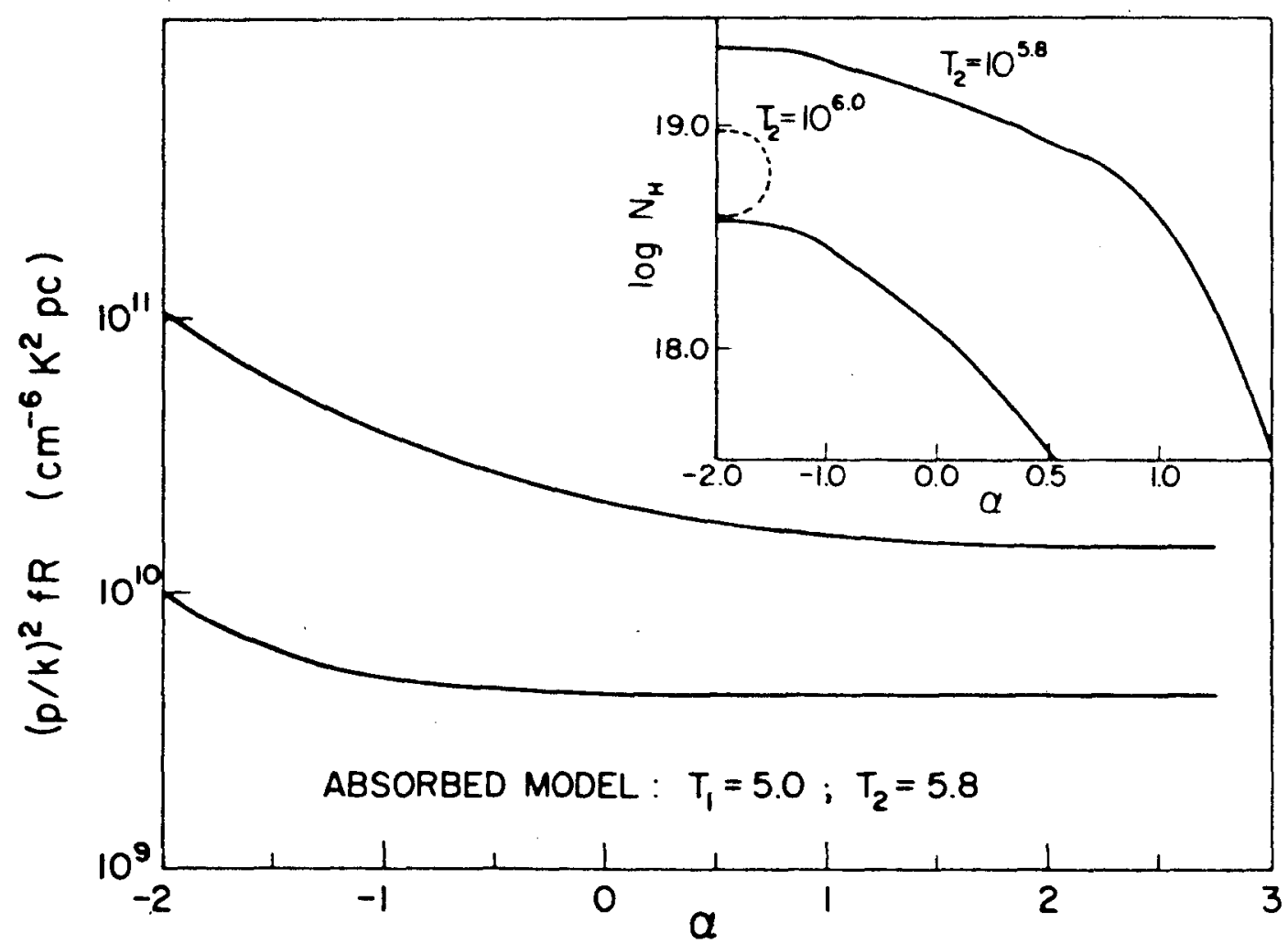

Figure 6 